\title{
Novel enhancement mechanisms of the nociceptive response by serum exosomes in a mouse model of neuropathic pain
}

\author{
Kengo Hamamura ${ }^{1}$, Soh Katsuyama ${ }^{2}$, Yohei Toyofuku ${ }^{1}$, Shunpei Watanabe ${ }^{1}$, Yuri Nishimura ${ }^{1}$, \\ Nanami Sueishi ${ }^{1}$, Taisei Tanaka ${ }^{1}$, Takaaki Komatsu ${ }^{3}$, Tsukasa Sakurada ${ }^{4}$, Kosuke Aritake ${ }^{1}$ \\ ${ }^{I}$ Department of Chemical Pharmacology, Daiichi College of Pharmaceutical Sciences, Japan, ${ }^{2}$ Center for Education of \\ Clinical Pharmacy, Tokyo University of Pharmacy and Life Sciences, Japan, ${ }^{3}$ Drug analysis laboratory, Daiichi \\ College of Pharmaceutical Sciences, Japan, ${ }^{4}$ Center for Supporting Pharmaceutical Education, Daiichi College of \\ Pharmaceutical Sciences, Japan
}

$<$ Background $>$ Exosomes are small $(40-150 \mathrm{~nm})$ membrane vesicles of endocytic origin that are found in many bodying fluids including blood and urine, and supporting their role in intercellular communication. Although recent studies have demonstrated that various biomarkers involved in the extent of pain from the serum exosomes, the effects of exosomes on the onset and progress of pain have not been elucidated. The objective of this study was to identify the relationship between serum exosomes in mice with partial sciatic nerve ligation (PSL) and alterations of nociceptive responses induced by $0.5 \%$ formalin.

$<$ Methods $>$ Ligation of partial sciatic nerve was performed in four-week-old male ddY-strain mice. A sciatic nerve was exposed through on incision and half of the nerve was ligated with surgical suture. Exosomal fraction in serum on day 7 post-PSL was isolated by ultracentrifugation. Exosomes were administered by intrathecally (i.t.) at the level of the 5th or 6th vertebrae of normal mice. $0.5 \%$ formalin was injected subcutaneous (s.c.) into the plantar surface of the right hindpaw just after i.t. exosomes injection. Each mouse was immediately returned to the observation chamber after formalin injection. Licking and biting of the injected hindpaw were defined as a nociceptive response, and the summation of time spent licking and biting was measured for $5 \mathrm{~min}$ after the formalin injection.

$<$ Results $>$ We have confirmed that the i.t. injection of serum exosomes from PSL mice or sham-operated mice transferred into the normal mice did not show any spontaneous nociceptive responses such as licking/biting or scratching. However, $0.5 \%$ formalin-induced nociceptive response was significantly enhanced by i.t. pretreatment with serum exosomes isolated from PSL mice but not from sham-operated mice. In addition, we treated the exosomes isolated from PSL by trypsin to obtain the "surface protein shaved" exosomes. The surface protein shaved PSL exosomes were ineffective on formalininduced response.

$<$ Conclusion $>$ Our data indicate that the surface protein of exosomes in mice with PSL may play an important role in enhancing nociceptive responses. 